\title{
UPTAKE OF PASSIVE IMMUNITY BY THE COMPROMIZED NEWBORN ANIMAL
}

\author{
Per T Sangild
}

Animal Nutrition and Reproduction, Royal Veterinary and Agricultural University, Frederiksberg C, DK-1870, Denmark

Circulating immunoglobulins constitute a key element in the host defence against environmental antigens. Before birth, the fetus is well protected from antigens by the protective barrier of the placenta. Just after birth, the newborn must be able to respond immunologically to a massive invasion of potentially harmful antigens and microorganisms from the surrounding environment. However, the innate immune defence is immature at the time of birth and some passive immunization from the mother is required until an active immune system is developed. Humans and certain other species are born with passive immunity in the form of maternal immunoglobulins transferred across the placenta before birth. In the large farm animals, the placenta does not allow transfer of maternal immunoglobulins before birth. The newborns from these species therefore depend entirely on the transmission of immunoglobulins from mother's colostrum transported across the small intestine immediately after birth. In the large farm animals, intestinal immunoglobulin absorption occurs mainly by a non-specific endocytosis of macromolecules, but the details of the absorption process and its cessation after 1-2 days of colostrum exposure ('intestinal closure') remains poorly understood. Little is known about both the feed and animal factors affecting the macromolecule absorptive capacity of the small intestine. This knowledge is important because a poor absorption of immunoglobulins from colostrum is known to increase neonatal disease susceptibility and mortality. The aim of this review is to provide information about the ability of the 'compromised newborn farm animal' to absorp colostral immunoglobulins. Stressful delivery, premature birth, fetal growth retardation and in vitro embryo production are factors that potentially lead to a compromised newborn animal. The available literature indicates that the ability of the small intestine to absorb macromolecules is severely reduced in response to premature birth and also negatively affected by some forms of metabolic and environmental stress after birth. The ability to take up immunoglobulins is also severely impaired, in both premature and term animals, if immunoglobulins are absorbed from diets other than mother's colostrum. On the other hand, fetal growth retardation, in vitro embryo production, or the birth process does not reduce the ability of the newborn intestine to absorb immunoglobulins from colostrum. Knowledge about both the diet- and animal-related factors which influence intestinal immunoglobulin uptake is important to improve the clinical care for both the normal and the 'compromized' newborn farm animal.

\section{COLOSTRUM - MORE THAN JUST AN IMMUNOGLOBULIN SUPPLIER}

\section{J.W. Blum}

Division of Nutrition and Physiology, Institute of Animal Genetics, Nutrition and Housing, University of Berne, CH-3012 Berne, Switzerland.

The adaptation of neonates to various environmental factors is critical and usually associated with the highest morbidity and mortality rates in the lifespan of farm animals. Adaptability is basically 
hampered by immaturity of organ functions and controlling systems. Thus, the growth hormone - insulin-like growth factor (IGF) axis, which is of primary importance for postnatal growth regulation, is not fully functioning. Some changes are primarily of constitutional nature. Thus, in young calves plasma nitrate and creatinine concentrations decrease from high concentrations at birth during the first week of life to the values seen in older cattle and can not be influenced by nutrition. Major changes occur with respect to nutrition because the primarily parenteral supply of nutrients changes to an exclusively enteral supply. The proper functioning of the gastrointestinal tract (GIT) is therefore of primary importance and characterized by exquisite developmental and functional changes. The intake of colostrum (C) by newborn calves not only influences the immune status, but also the development of the GIT and of other organs, metabolism and endocrine systems. C contains high amounts of cells (leucocytes), nutrients (carbohydrates, especially lactose; fatty acids and lipids; amino acids and proteins; minerals; vitamins), bioactive substances (hormones, growth factors and cytokines, such as insulin-like growth factors, epidermal growth factor, insulin, prolactin and tumor necrosis factor- $\alpha$; enzymes; nucleotides; polyamines). The greatest mass of these nutrient and nonnutrient substances - with the major exception of casein and lactose - is available to newborns with the first $\mathrm{C}$. Ingestion of $\mathrm{C}$ is essential for all farm animals. $\mathrm{C}$ intake (as shown in calves) stimulates the proliferation of the small intestine, increases villous size and absorptive capacity, an effect which is likely associated with reduced apoptosis. As shown in calves, there are specific receptors for several of the ingested hormones and growth factors (such as insulin, IGFs) localized in the mucosa of the small intestine and colon. The receptor number is exhibiting ontogenetical changes and/or is influenced by early postnatal nutrition and it can basically be expected that the ingested hormones and growth factors influence GIT development upon receptor interaction. Feeding IGF-I alone to calves did, however, not influence small intestinal epithelial proliferation and villous size, but feeding an extract derived from first-milked bovine $\mathrm{C}$ (which contained high amounts of IGFs, insulin, lactoferrin and various other growth factors) to a formula containing nearly no bioactive substances stimulated small intestinal epithelial proliferation and villous size. The intestinal absorption of immunoglobulins and of some other proteins and peptides is possible only during a short period after birth (length depending on species) and there is indication that (at least in calves) the status of non-esterified fatty acids, triglycerides, phospholipids, essential fatty acids, $\beta$-carotin, retinol and tocopherol depends, too, on early postnatal $\mathrm{C}$ ingestion. In addition, the plasma amino acid profile and especially the glutamine/glutamate ratio (in calves) depends on whether $\mathrm{C}$ is ingested immediately after birth or not. Even though the intake of lactose is smaller than if mature milk is ingested, early $\mathrm{C}$ intake is also important for longer-lasting glucose homeostasis, likely due to enhanced gluconeogenesis. Time-point and amount of $\mathrm{C}$ intake influence the endocrine status, such as (in calves) plasma concentrations of gastrin, glucose-dependent insulinotropic hormone, insulin, glucagon, IGF-I, IGF binding proteins, and cortisol. Optimal amounts of $\mathrm{C}$ to be provided to newborns are not clearly defined, but ad libitum availability of $\mathrm{C}$ appears most appropriate, as is the case when neonates are allowed to suckle on their dam. Because calves in the first week of life under ad libitum (automate) feeding conditions ingest up to $11 \mathrm{~L} \mathrm{C/d}$ from $\mathrm{d} 2$ on, recommendations may have to be changed. In conclusion, the sum of all colostral factors is of importance, although some of the factors ingested with $\mathrm{C}$ are of special importance for an appropriate nutritional and metabolic status. These changes observed in association with feeding especially of $\mathrm{C}$ are important for the critical first week(s) of life. 